CLINICAL STUDY

\title{
Assessment of androgen concentration in women: liquid chromatography-tandem mass spectrometry and extraction RIA show comparable results
}

\author{
Femi Janse $^{1}$, Martinus J C Eijkemans ${ }^{1,2}$, Angelique J Goverde ${ }^{1}$, Eef G W M Lentjes ${ }^{3}$, Annemieke Hoek ${ }^{4}$, \\ Cornelius B Lambalk ${ }^{5}$, Theresa E Hickey ${ }^{6}$, Bart C J M Fauser ${ }^{1}$ and Robert J Norman ${ }^{6}$ \\ ${ }^{1}$ Department of Reproductive Medicine and Gynecology, University Medical Center Utrecht, Room F05-126, PO Box 75500, 3508 GA Utrecht, The \\ Netherlands, ${ }^{2}$ Julius Center for Health Sciences and Primary Care and ${ }^{3}$ Laboratory of Endocrinology, Department of Clinical Chemistry and Hematology, \\ University Medical Center Utrecht, Utrecht, The Netherlands, ${ }^{4}$ Section of Reproductive Medicine, Department of Obstetrics and Gynecology, University \\ Medical Center Groningen, University of Groningen, Groningen, The Netherlands, ${ }^{5}$ Department of Reproductive Medicine, VU University Medical Center, \\ Amsterdam, The Netherlands and ${ }^{6}$ Discipline of Obstetrics and Gynaecology, School of Paediatrics and Reproductive Health, Robinson Institute, Research \\ Centre for Reproductive Health, University of Adelaide, Adelaide, South Australia, Australia
}

(Correspondence should be addressed to F Janse; Email: f.janse@umcutrecht.nl)

\begin{abstract}
Objective: The measurement of serum testosterone in women is challenging due to lack of trueness, precision, and sensitivity of various available testosterone assays. Accurate assessment of testosterone in women is crucial especially in conditions associated with alleged over- or under-production of testosterone, such as in polycystic ovary syndrome (PCOS) or primary ovarian insufficiency (POI). The aim of this study was to measure and compare androgen concentrations in women with PCOS, POI, and female controls and to evaluate the performance of extraction RIA and liquid chromatographytandem mass spectrometry (LC-MS/MS) in these women.

Design: Cross-sectional study.

Methods: Carefully phenotyped women with POI $(n=208)$ or PCOS $(n=200)$ and 45 healthy, regularly cyclic female controls were included. Method comparison analyses were performed for total testosterone, androstenedione (AD), and DHEA, as measured by LC-MS/MS and extraction RIA. Results: All androgen levels were significantly elevated in women with PCOS compared with POI patients $(P<0.05)$ and controls $(P<0.05)$. Women with POI presented with similar androgen concentrations as controls, except for AD. Compared with measurements by extraction RIA, testosterone, DHEA, and AD concentrations measured by LC-MS/MS were systematically lower. However, using extraction RIA and LC-MS/MS, testosterone, DHEA, and AD measurements were shown to have good agreement as assessed by Bland-Altman analysis and intraclass correlation coefficient: 0.95 (95\% confidence interval 0.94-0.91), $0.83(0.79-0.86)$, and 0.96 (0.95-0.97) respectively.

Conclusions: LC-MS/MS, compared with a labor-intensive extraction RIA, shows good precision, sensitivity, and high accuracy for measuring female testosterone, DHEA, and AD concentrations under various clinical conditions. LC-MS/MS, therefore, represents a convenient and reliable assay for both clinical and research purposes, where androgen measurement in women is required.
\end{abstract}

European Journal of Endocrinology $165925-933$

\section{Introduction}

The measurement of serum testosterone has proven to be a great challenge due to lack of precision and sensitivity of various commercially available testosterone assays, resulting in limited utility (1). Testosterone assays were originally developed to measure testosterone concentrations in the normal male range (2). However, women exhibit testosterone levels well below this range, and therefore, reliable measurement of female testosterone concentrations is particularly problematic due to trueness and precision problems (3).
In women of reproductive age, $25 \%$ of testosterone biosynthesis occurs in the ovarian theca cells. Another $25 \%$ is produced by the adrenal gland, and the remaining $50 \%$ of circulating testosterone derives from peripheral conversion of testosterone precursors (DHEA and androstenedione (AD)). DHEA and its sulfate metabolite (DHEAS) are exclusively produced by the adrenal glands, whereas AD is produced by both the adrenal and the ovary $(4,5)$. Most circulating testosterone is biologically inactive as it binds to serum proteins, primarily sex hormone-binding protein (SHBG) and albumin $(2,6)$. 
The most widely used methods for measuring serum testosterone are the simple, relatively inexpensive RIA and chemiluminescence immunoassay, performed directly in serum. Although the immunoassays show average good precision, they often show more bias. This is especially true for the lower range, where they can be subject to increased interference and overestimation of steroid concentrations compared with other assays $(7,8)$. Using extraction and chromatography methods preceding RIA has the advantage of removing interfering proteins and cross-reacting steroids. Although extraction RIA seems preferable over direct assays, it is infrequently used in clinical practice because proper validation is lacking and extraction is labor intensive and time consuming $(1,9,10)$. A commonly used practical approach to estimate bioactive testosterone is the calculation of the free androgen index (FAI). While FAI was shown to correlate quite well with physical separation measures of female free testosterone (not male), FAI is highly dependent on the quality of testosterone and SHBG assay measurements (2).

In order to overcome these difficulties, tandem mass spectrometry (MS/MS) preceded by gas or liquid chromatography (GC-MS/MS and LC-MS/MS) assays for steroid measurement is emerging (11). LC-MS/MS has some advantages over the use of immunoassays, especially compared with those performed on platforms. LC-MS/MS shows equal or better precision, and these assays do not suffer from interferences due to chromatographic separation and mass spectrometry analysis. Interferences can cause serious bias at low testosterone concentrations, which may be encountered in postmenopausal women and children. However, the high costs of mass spectrometry equipment may be a possible drawback for routine clinical use. Although LC-MS/MS assays show higher precision and better accuracy than immunoassays, there is still a difference in performance (sensitivity, specificity, and trueness) among LC-MS/MS methods and thus there is a need for standardization (12).

Reliable assessment of testosterone in women is crucial for the diagnosis of conditions associated with alleged overproduction of testosterone such as in polycystic ovary syndrome (PCOS), or conditions that may incur androgen deficiency, such as postmenopause, oophorectomy, or primary ovarian insufficiency (POI) (13-15). However, analysis of serum testosterone by commercial assays fails to identify biochemical hyperandrogenism in $20-40 \%$ of women with PCOS, while hyperandrogenism is one of the criteria on which the diagnosis is based $(16,17)$. Moreover, female androgen insufficiency remains controversial due to the lack of reliable serum testosterone assays to define this condition (10). Clinical guidelines concerning indications for androgen therapy in case of hypoandrogenism cannot be established (18). These examples illustrate the need for a reliable testosterone measurement, which is easily available at reasonable cost for both clinical and research purposes.
The current study was aimed at evaluating the measurement of testosterone and other androgens by LC-MS/MS in comparison to measurement by extraction RIA in women with normal and presumably elevated and decreased androgens.

\section{Materials and methods}

\section{Subjects}

In 2004, a study involving standardized phenotyping and follow-up was initiated in the University Medical Center Utrecht, The Netherlands, in which all women suspected of menstrual cycle disturbance and referred to our outpatient clinic participated. Details of the systematic evaluation, approved by the local ethics committee, have been described previously $(19,20)$. For the current study, 169 women with POI and 200 women affected by PCOS, who visited the outpatient clinic until July 2009, were included. POI was defined as secondary amenorrhea for at least 4 months in women younger than 40 years, along with repeated FSH concentrations exceeding $40 \mathrm{IU} / \mathrm{l}$ (21). PCOS was diagnosed according to the Rotterdam consensus criteria if at least two of the following criteria were present: i) oligo/anovulation, ii) clinical and/or biochemical hyperandrogenism (testosterone $>2.0 \mathrm{nmol} / \mathrm{l}$, assessed by our in-house extraction RIA), and/or iii) polycystic ovaries on ultrasonography (17). Additionally, 39 women with POI visiting four other centers (participating in the Dutch POI Consortium $(20,22)$ ) were also included in the current study.

As controls, 45 women visiting the preconceptional clinic prior to starting IVF/ICSI treatment because of severe male infertility between October 2006 and July 2008 were included. Severe male infertility was defined as semen analysis with volume $\times$ concentration $\times$ motility below 2.0 million. All women were healthy and experienced regular menstrual cycles. Exclusion criteria were PCOS, previous poor response after ovarian hyperstimulation, age $>38$ years, early follicular FSH concentrations $>12 \mathrm{U} / \mathrm{l}$, and thyroid dysfunction. All included study subjects gave written informed consent.

\section{Measurements}

Serum samples were collected during the early follicular phase of the menstrual cycle in women with regular cycles. In case of a cycle disturbance, samples were drawn at a random cycle day and a progesterone concentration was measured concomitantly to ensure preovulatory state. None of the women had used any hormonal medication for at least 2 weeks. After collection and aliquoting, one fresh sample was analyzed immediately while the remaining aliquots were stored at $-20{ }^{\circ} \mathrm{C}$. In Utrecht, the fresh testosterone measurement was performed using an in-house 
extraction RIA, after diethylether extraction of $500 \mu \mathrm{l}$ sample, using a polyclonal anti-testosterone antibody (Dr Pratt AZG 3290, Department of Nuclear Medicine, University Medical Center Groningen). (1,2- $\left.{ }^{3} \mathrm{H}(\mathrm{N})\right)$ testosterone (NET-387, DuPont NEN B.V., Dordrecht, The Netherlands) was used as a tracer following chromatographic verification of its purity. A small amount of recovery tracer was added to the sample to correct for losses during extraction. The lower limit of quantification (LLQ), i.e. the concentration that can be determined with $20 \%$ coefficient of variation $(\mathrm{CV}$; estimated from a precision profile), was $0.10 \mathrm{nmol} / \mathrm{l}$ and inter-assay variation was 10,6 , and $7 \%$ at 0.85 , 2.6 , and $12 \mathrm{nmol} / \mathrm{l}$ respectively $(n=100)$. Intra-assay CVs at 2 and $10 \mathrm{nmol} / \mathrm{l}$ were 3.8 and $3.5 \%$ respectively. There was no interference detected from dihydrotestosterone, $\mathrm{AD}$, and DHEA. Recent comparison of the in-house method with a dilution liquid (ID)-GC/MS method using 20 human serum samples of the Dutch EQAS gave the following correlation: RIA $=1.13 \times(\mathrm{ID}-$ $\mathrm{GC} / \mathrm{MS}$ ) $+0.33 \mathrm{nmol} / \mathrm{l}$ (tested range was $0.8-33 \mathrm{nmol} / \mathrm{l}$; 95\% confidence limit of the slope: $1.04-1.20$ and of the intercept: -1.04 to 0.91) (23). Furthermore, the following measurements were performed on fresh serum for women with POI and PCOS: estradiol $\left(\mathrm{E}_{2}\right)$, SHBG, DHEA, DHEAS, and AD. $E_{2}$ concentrations were measured using the Roche E170 Modular (Roche). SHBG concentrations were quantified using an Immulite platform (Diagnostic Products Corporation, Breda, The Netherlands). DHEA was measured after diethylether extraction and Celite chromatography using an in house RIA. DHEAS was measured using the Coat-A-Count DHEA-SO $_{4}$ RIA (Siemens Diagnostics, Breda, The Netherlands). AD was measured after hexane-toluene extraction using an in-house RIA. Interassay CVs for $\mathrm{E}_{2}$ were $13,5.7$, and $3.4 \%$ at 66,200 , and $600 \mathrm{pmol} / \mathrm{l}$ $(n=100)$ respectively; for SHBG $4.2,3.9$, and $4.4 \%$ at 10,36 , and $120 \mathrm{nmol} / \mathrm{l}(n=93)$ respectively; for DHEA $7.7,7.6$, and $8.8 \%$ at $1.4,4.2$, and $12.5 \mathrm{nmol} / \mathrm{l}(n=29)$ respectively; for DHEAS $8.5,5.5$, and $6.8 \%$ at $1.3,5.3$, and $12.8 \mu \mathrm{mol} / \mathrm{l}(n=60)$ respectively; and for AD 11.5, 5.7 , and $6.7 \%$ at 1,5 and $11 \mathrm{nmol} / \mathrm{l} \quad(n=50)$ respectively. Intra-assay CVs for DHEA were 9 and $5 \%$ at 5 and $20 \mathrm{nmol} / \mathrm{l}$, respectively; for $\mathrm{AD}$, it was $4 \%$ at $7 \mathrm{nmol} / \mathrm{l}$. For $\mathrm{E}_{2}$, DHEAS, and SHBG, the intra-assay CVs were within the assay documentation. Testosterone, $\mathrm{AD}$, and DHEA were calibrated against gravimetrically determined quantities of pure standard in steroid-free BSA. For the other assays, we used the calibration of the immunoanalyzers. All measurements were performed in duplicate. The reference range for cycling women in this laboratory were the following: for testosterone: $0.50-2.00 \mathrm{nmol} / \mathrm{l}$, for DHEA 10-30 nmol/l, and for AD: 3.0-7.0 nmol/l. Serum SHBG concentrations were also measured in female controls, using identical assays.

Testosterone, AD, and DHEA were also measured in each individual sample at the CPR Pharma Pty Ltd
Laboratory, organized by the University of Adelaide, Australia. Frozen aliquots were sent to Australia and thawed just before use. Testosterone, AD, DHEA, internal standards ( $\mathrm{d}_{3}$-testosterone (IS-testosterone), and $19-\mathrm{d}_{3}$-androstenedione (IS-AD)) were extracted using liquid-liquid extraction with hexane-dichloromethane solvent mixture. The analytes were separated by HPLC on an Alltima HP C18HL column, and the eluates were monitored by an API5500 MS/MS detector in positive multiple reaction monitoring (MRM) mode. The single charged $Q 1 / \mathrm{Q} 3$ transition is 289.3/96.9, 287.1/97.0, and 289.2/253.2 amu for testosterone, AD, and DHEA, respectively, and 292.2/97.0 and 290.2/100.0 amu for IS-testosterone and IS-AD respectively (see Supplementary Figure 1, see section on supplementary data given at the end of this article). The extract was then assayed against a calibration curve, and data were acquired and processed by the data acquisition system, Analyst 1.5 linked directly to the API5500 MS/MS detector. The method's detection range is from 0.175 to $69.6 \mathrm{nmol} / \mathrm{l}$ for testosterone and AD and 4.34-1740 nmol/l for DHEA. Measurements were performed in duplicate. Accuracy for testosterone against nine validated pure standards was assessed as above and ranged from -2.0 to $+3.8 \%$ across the complete standard range (eight replicates) and between -2.0 and $+1.6 \%$ in the range $0.173-$ $3.47 \mathrm{nmol} / \mathrm{l}$. Precision for testosterone ranged between 1.5 and $7.5 \%$ across the whole range and was $1.5-9.3 \%$ between 0.173 and $3.47 \mathrm{nmol} / \mathrm{l}$. Results were similar for all analytes measured. The following inter-assay CVs were identified: in controls: $5.6 \%$ (testosterone), $8.4 \%$ (DHEA), and 6.4\% (AD); in PCOS: 4.3\% (testosterone), $5.9 \%$ (DHEA), and $4.5 \%$ (AD). The limit of detection was determined by repeated measures of the three lowest standards used in the assay. A precision dose profile was established and statistical significance between the standards shown by clear separation of the doses. The run time was $10 \mathrm{~min}$ per sample.

FAI was calculated for both testosterone measurements: testosterone/SHBG $\times 100$ (24) using the SHBG measurement performed in Utrecht.

\section{Statistical analysis}

Median values and range for each individual group of women were compared using one-way analysis of covariance and Bonferroni post-hoc tests for significance, correcting for age and body mass index (BMI; possible confounders) (25). Method comparison analyses were performed by constructing scatter plots and Bland-Altman plots to evaluate the extent to which testosterone, DHEA, and AD measurements agreed in both methods. Bland-Altman plots show the percentage difference between two corresponding measurements plotted against the measurements' mean.

For all Bland-Altman plots, the mean percentage difference between the methods (mean bias) with 
associated 95\% confidence intervals (CIs) and limits of agreement ( \pm 2 s.D.) were calculated. Intraclass correlation coefficients (ICC) with 95\% CIs were calculated for testosterone, DHEA, and AD. Large ICC (maximum 1) indicates that variation between both assays is equal to zero (perfect agreement) $(26,27)$. Because androgen concentrations were not normally distributed, logarithmic transformation of variables was applied before execution of analyses. When testosterone or DHEA concentrations were undetectable, $0.5 \times$ LLOD was used (28).

To further compare the performance of extraction RIA and LC-MS/MS for the measurement of testosterone, DHEA, and AD in this population, correlations between various androgens and $\mathrm{E}_{2}$ were assessed. To this end, linear regression analyses were performed, where testosterone (RIA) and testosterone (LC-MS/MS) were entered separately and similarly repeated, entering the variables in the opposite order. Univariate standardized $\beta$ coefficients with associated S.E.M. are reported. Larger univariate standardized $\beta$ coefficients represent stronger associations with the steroids. Univariate standardized $\beta$ coefficients of both testosterone assays were compared using a bootstrapping procedure with 2000 replications, because both testosterone measurements were performed in identical samples. 95\% CIs were calculated to identify significant differences between the correlations with androgens and $E_{2}$ vs the testosterone measurements by RIA and LC-MS/MS assays. Identical analyses were performed for DHEA (RIA) and DHEA (LC-MS/MS), and for AD (RIA) and AD (LC-MS/MS). Statistical analyses were performed using SPSS 15.0 (SPSS, Inc., Chicago, IL, USA) and R version 2.9.0. (http://www.r-project.org/).

\section{Results}

Median concentrations of all measurements are shown per patient group in Table 1 . In women with PCOS, all hormone concentrations were significantly higher compared with those in POI patients and in controls, with exception of SHBG. Androgens (except $\mathrm{AD}), \mathrm{E}_{2}$, and SHBG concentrations were similar for women with POI and controls.

The scatter and Bland-Altman plots for testosterone, DHEA, and $\mathrm{AD}$ measurements are depicted in Figs 1-3. All scatter plots show high correlation between extraction RIA and LC-MS/MS with correlation coefficients of 0.960 for testosterone and $\mathrm{AD}$ and 0.830 for DHEA. The ICC for testosterone, DHEA, and AD were 0.95 (95\% CI 0.94-0.96), 0.83 (0.79-0.86), and 0.96 (0.95-0.97), respectively, indicating good agreement between both extraction RIA and LC-MS/MS assays. It becomes evident from all Bland-Altman plots that the average percent difference (i.e. mean bias) in serum testosterone, DHEA, and AD levels between LC-MS/MS and extraction RIA were $-31.5,-7.1$, and $-20.9 \%$ respectively. This indicates that LC-MS/MS yields significantly lower serum testosterone and AD results than extraction RIA, which may possibly be explained by interfering substances in the latter assay. In $24(5.3 \%)$ of testosterone, $22(6.0 \%)$ of DHEA, and $16(4.4 \%)$ of AD, the differences between the methods were beyond the limits of agreement (mean difference \pm 2 S.D.), with the majority of these outliers occurring in the lower concentration ranges. Small, insignificant differences may incur a great percentage difference when calculated in the low range, which is dramatically shown in a Bland-Altman plot.

Table 1 Baseline characteristics for each patient group. Results are shown as median (range). Analysis of covariance was carried out on logarithmically transformed variables to study the differences between patient groups.

\begin{tabular}{|c|c|c|c|c|}
\hline & Controls $(n=45)$ & POI $(n=208)$ & $\operatorname{PCOS}(n=200)$ & $\boldsymbol{P}$ \\
\hline Age (years) & $33.1(20.3-37.8)$ & $37.6(15.8-62.9)$ & $29.3(17.9-41.0)$ & $<0.001$ \\
\hline $\mathrm{BMI}\left(\mathrm{kg} / \mathrm{m}^{2}\right)$ & $23.4(17.1-36.9)$ & $23.4(17.1-37.3)$ & $24.2(16.2-55.9)$ & 0.003 \\
\hline Testosterone RIA (nmol/l) & $1.20(0.61-3.90)$ & $1.10(0.29-3.00)$ & $2.00(0.64-6.30)$ & $<0.001$ \\
\hline Testosterone LC-MS/MS $(\mathrm{nmol} / \mathrm{l})^{\mathrm{a}}$ & $0.87(0.31-2.78)$ & $0.76(0.09-2.30)$ & $1.55(0.34-5.48)$ & $<0.001$ \\
\hline $\mathrm{E}_{2} \mathrm{RIA}(\mathrm{pmol} / \mathrm{l})$ & N/A & $40(20-1500)^{b}$ & $200(70-2250)$ & $<0.001$ \\
\hline SHBG RIA (nmol/l) & $60(12-199)$ & $55(17-201)$ & $47(7-191)$ & $0.04^{*}$ \\
\hline DHEA RIA $(\mathrm{nmol} / \mathrm{l})$ & $\mathrm{N} / \mathrm{A}$ & $11.0(1.10-43.0)^{\mathrm{c}}$ & $18.0(2.00-67.0)$ & 0.02 \\
\hline DHEA LC-MS/MS $(\mathrm{nmol} / /)^{d}$ & $16.0(8.74-58.9)$ & $12.1(4.34-43.0)$ & $15.8(4.34-65.2)$ & NS \\
\hline DHEAS RIA $(\mu \mathrm{mol} / \mathrm{l})$ & N/A & $3.90(0.41-12.0)^{\mathrm{c}}$ & $4.80(0.77-14.0)$ & NS \\
\hline AD RIA (nmol/l) & $\mathrm{N} / \mathrm{A}$ & $2.90(0.80-8.90)^{\mathrm{e}}$ & $7.20(2.40-22.0)$ & $<0.001$ \\
\hline AD LC-MS/MS (nmol/l) & $3.29(1.31-10.9)$ & $2.23(0.33-8.46)$ & $5.87(1.81-15.9)$ & $<0.005$ \\
\hline FAI RIA & $1.83(0.75-15.8)$ & $1.76(0.29-8.24)$ & $4.65(0.68-26.2)$ & $<0.001$ \\
\hline FAI LC-MS/MS & $1.29(0.48-10.7)$ & $0.56(0.02-2.11)$ & $3.36(0.60-21.0)$ & $<0.001$ \\
\hline
\end{tabular}

All $P$ values are adjusted for age and BMI. Italic values in $P$ column indicate that all groups were significantly different from each other; bold values in $P$ column indicate that PCOS was significantly different from controls and POI; ${ }^{*}$, indicates that PCOS was significantly different from controls. N/A, not available;

NS, not significant; LLOD, lower limit of detection.

aMeasurements below LLOD: in POI, $n=1(0.4 \%)$.

${ }^{\mathrm{b}} n=195$.

${ }^{\mathrm{c}} n=162$.

${ }^{\mathrm{d}}$ Measurements below LLOD: in controls, $n=15$ (33\%); POI, $n=58(28 \%)$; and PCOS, $n=14(7 \%)$.

${ }^{\mathrm{e}} n=164$. 
A
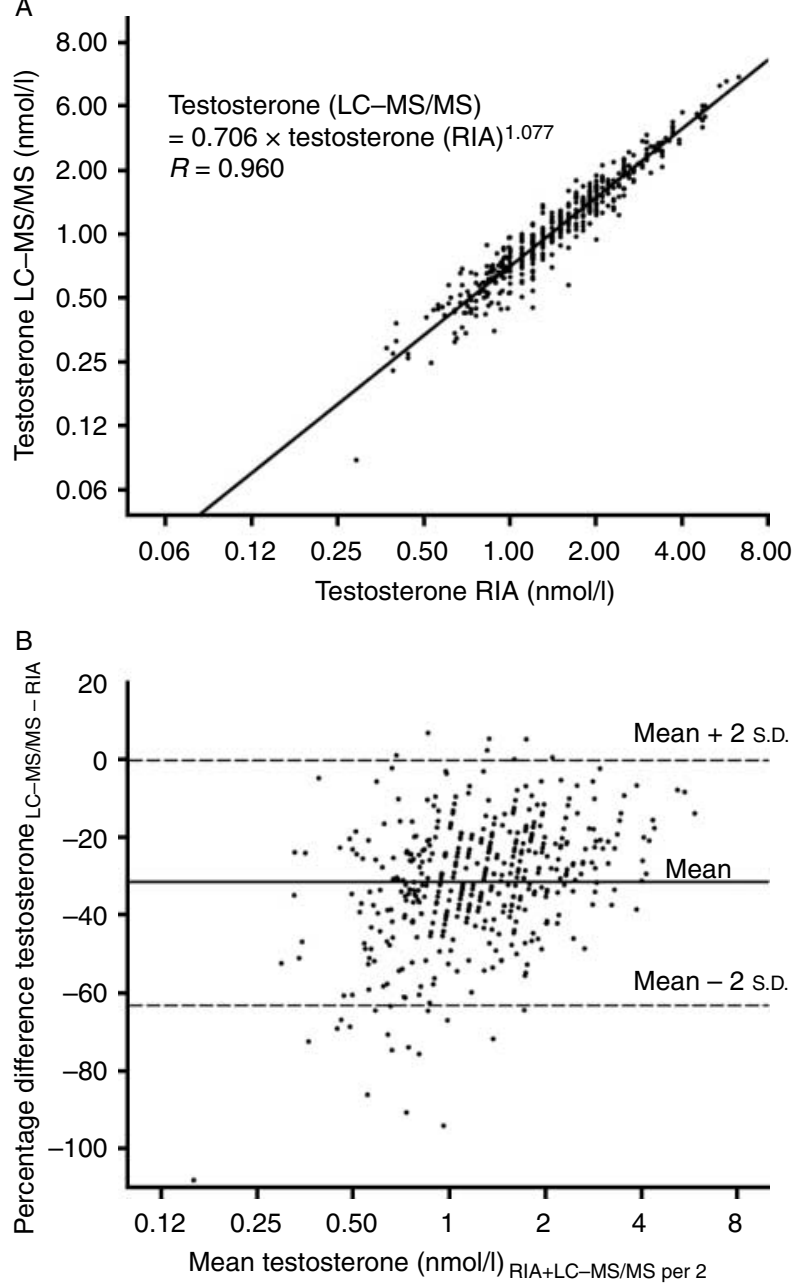

Figure 1 Scatter plot $(A)$ and Bland-Altman plot $(B)$ to show the extent to which testosterone measurements agreed in both methods. The solid line represents the mean percentage difference between the methods (mean bias) and the dashed lines 2 s.D. of the mean percentage difference (limits of agreement). Filled circles represent individual measurements.

In conclusion, the Bland-Altman plots suggest that the two methods are in good agreement with testosterone, DHEA, and AD.

Finally, correlations between testosterone, DHEA, and $\mathrm{AD}$ vs related steroids (i.e. other androgens and $\mathrm{E}_{2}$ ) were calculated to identify whether the extraction RIA or the LC-MS/MS assays showed a difference in these associations (Supplementary Tables 1-3, see section on supplementary data given at the end of this article). In general, most correlations were similar among the assays. The identification of some small significant differences indicates that the strength of the association increased when related steroids were measured employing the identical assay with which the original measurement was performed.

\section{Discussion}

In this study, the performances of LC-MS/MS and an in-house extraction RIA were compared for a broad range of androgen concentrations encountered in women of reproductive age, including the clinical conditions POI and PCOS. LC-MS/MS and extraction RIA demonstrated good agreement with the measurement of testosterone, DHEA, and AD, although LC-MS/MS gave significantly lower concentrations when compared with extraction RIA. Furthermore, similar correlations were identified in comparison with the association for each assay with other steroids. Therefore, LC-MS/MS, compared with a labor-intensive extraction RIA, shows good precision, sensitivity, and high accuracy for the measurement of androgens in women with PCOS, POI, and regularly cycling female controls.

Androgen concentrations for women with PCOS were significantly higher compared with female controls and women affected by POI, which confirms other recent studies applying LC-MS/MS $(26,27)$. However, this is the first study to measure androgens with LC-MS/MS in women with POI. In this group, testosterone and DHEA concentrations were similar as female controls, while $\mathrm{AD}$ concentrations were significantly lower. In the existing literature, it is debated whether POI is associated with decreased androgen concentrations (14, 29-33), and only one study presented with results similar to ours (34). As previous studies only applied direct RIA, chemiluminescence immunoassays, and extraction RIA, conflicting findings most probably resulted from lower sensitivity, lack of specificity, or lower accuracy of these assays (10). While decline of androgen synthesis may result from menopausal status, this has also been associated with increasing age $(30,35)$. Therefore, the results of the current study support the concept that young women with POI encounter normal testosterone and DHEA concentrations, in contrast with women who experience menopause at a regular age. Currently, there is no explanation for the significantly lower $\mathrm{AD}$ concentrations encountered in women with POI. This finding needs further investigation.

MS after GC or LC is a relatively new technique for steroid sex hormone measurement and data on its applicability, particularly in the female sex hormone concentration range, are scarce. The LC-MS/MS assay in this study showed excellent precision and accuracy down to at least $0.173 \mathrm{nmol} / \mathrm{l}$ (precision $-0.2 \%$; accuracy $1.5 \%$ for authentic standards). This allows for a great deal of confidence in the reliability of an assay, which is completely specific for testosterone or other nominated steroid analytes. A study comparing LC-MS/MS with direct and extraction RIAs in normal and hypogonadal male subjects by Bland-Altman plots showed systematically lower results by LC-MS/MS (36). Studies on female and pediatric samples confirmed this finding, while also identifying that LC-MS/MS showed greater accuracy in lower androgen concentrations 


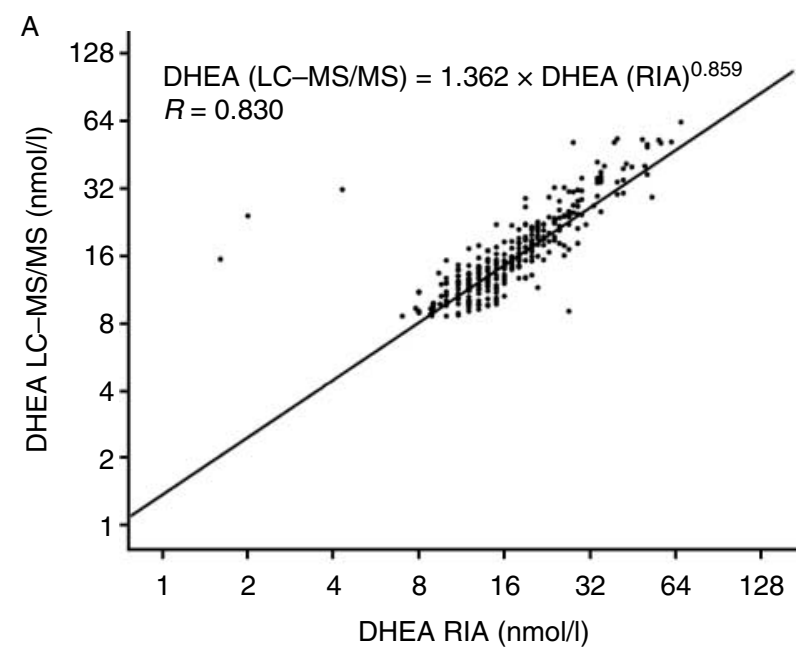

B

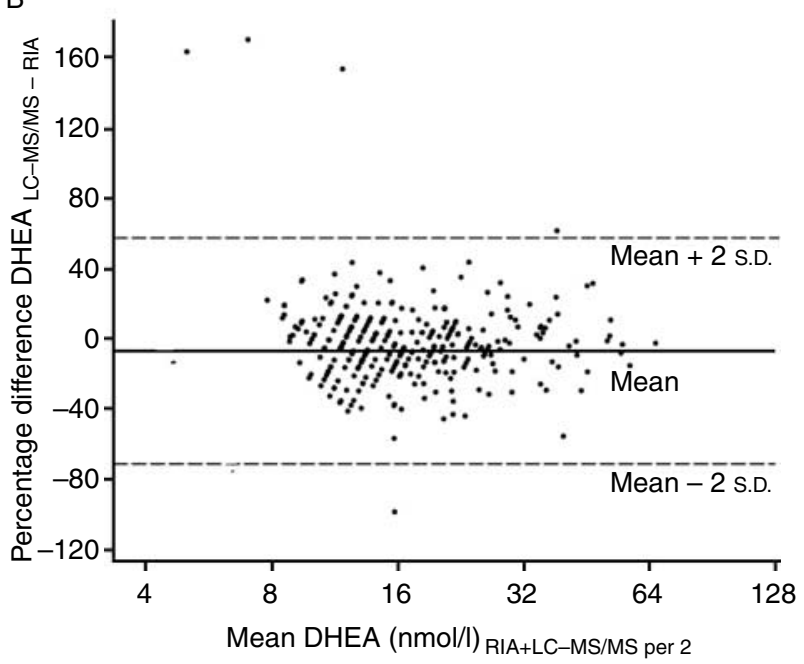

Figure 2 Scatter plot (A) and Bland-Altman plot (B) to show the extent to which DHEA measurements agreed in both methods. The solid line represents the mean percentage difference between the methods (mean bias) and the dashed lines 2 s.D. of the mean percentage difference (limits of agreement). Filled circles represent individual measurements.

than direct assays $(37,38)$. This study included serum samples of women with PCOS, POI, and normally cyclic women. We identified good agreement between LC-MS/MS and the in-house extraction RIA (even for lower ranges encountered in POI) and slightly lower hormone concentrations reported after LC-MS/MS. Previous studies on the comparison of LC-MS with extraction RIA identified overestimation of extraction RIA between 20 and $80 \%$ in testosterone concentrations below $3.47 \mathrm{nmol} / \mathrm{l}(1 \mathrm{ng} / \mathrm{dl})$, similar to our findings: between -0.2 and $63 \%$ (36). We are cautious to generalize the agreement between extracted RIA and LC-MS/MS in the current study, because only two centers participated in the current study. But the results are in accordance with previous studies $(36,37,39)$, including a study on women with PCOS, which confirms that well-chosen RIA may offer equal precision and accuracy like LC-MS/MS (40).

When examining the performance of extraction RIA and LC-MS/MS, it is important to include a clinical validation of the androgens measured by each assay. Ideally, correlation analyses should be performed with clear clinical endpoints, such as degree of hirsutism or presence of the female androgen deficiency syndrome. However, until now, clear clinical endpoints related to androgen concentrations cannot irrefutably be identified (40-42). Therefore, the associations of androgens, measured by both assays, were compared with other physiologically related steroids (androgens and $E_{2}$ ). Our results indicate that for the two assays evaluated in this study, the correlations were similar and both assays showed equal performance.

The major strength of this study is the large sample size of well-phenotyped women with PCOS and POI, representing the broad spectrum of female androgen concentrations during reproductive life. Laboratory personnel at both sites were blinded for study design and identity of the samples. But this study may also have potential weaknesses. While all extraction RIA assessments were done in fresh samples, frozen samples were shipped to Australia before LC-MS/MS processing. However, previous studies on long-term stored samples identified that steroids are very stable after being isolated in the serum (43). Furthermore, early follicular androgen concentrations in regularly cyclic controls may underrepresent androgen levels present during the middle third of the cycle (18). Finally, the group of female controls was relatively small, which may have concealed potential differences in androgen concentrations compared with those in women with POI.

Although our results show that LC-MS/MS is a reliable alternative for measuring steroid sex hormones in women, its introduction into clinical or research practice may depend on several factors: costs of the LC-MS equipment, LC-MS expertise in laboratories, and future RIA development. The LC-MS is now introduced in many laboratories, but the methods designed on this analyzer should meet the same requirements (defined by the FDA or European Directive) like the commercial immunoassays. This means that the validation of an LC-MS/MS procedure, like the validation of extraction RIAs, has to be much more extensive than for commercial immunoassays, because LC-MS assays are mostly in-house-developed methods unlike already validated kits. Furthermore, inappropriate sample preparation and analyte detection may greatly influence the performance of LC-MS/MS and extraction RIA procedures, thus emphasizing the importance of validation. Extraction RIA may gain increased specificity if more specific antibodies are selected, and the method for the displacement of bound testosterone from its binding proteins is optimized (36). If this occurs, new comparison studies 

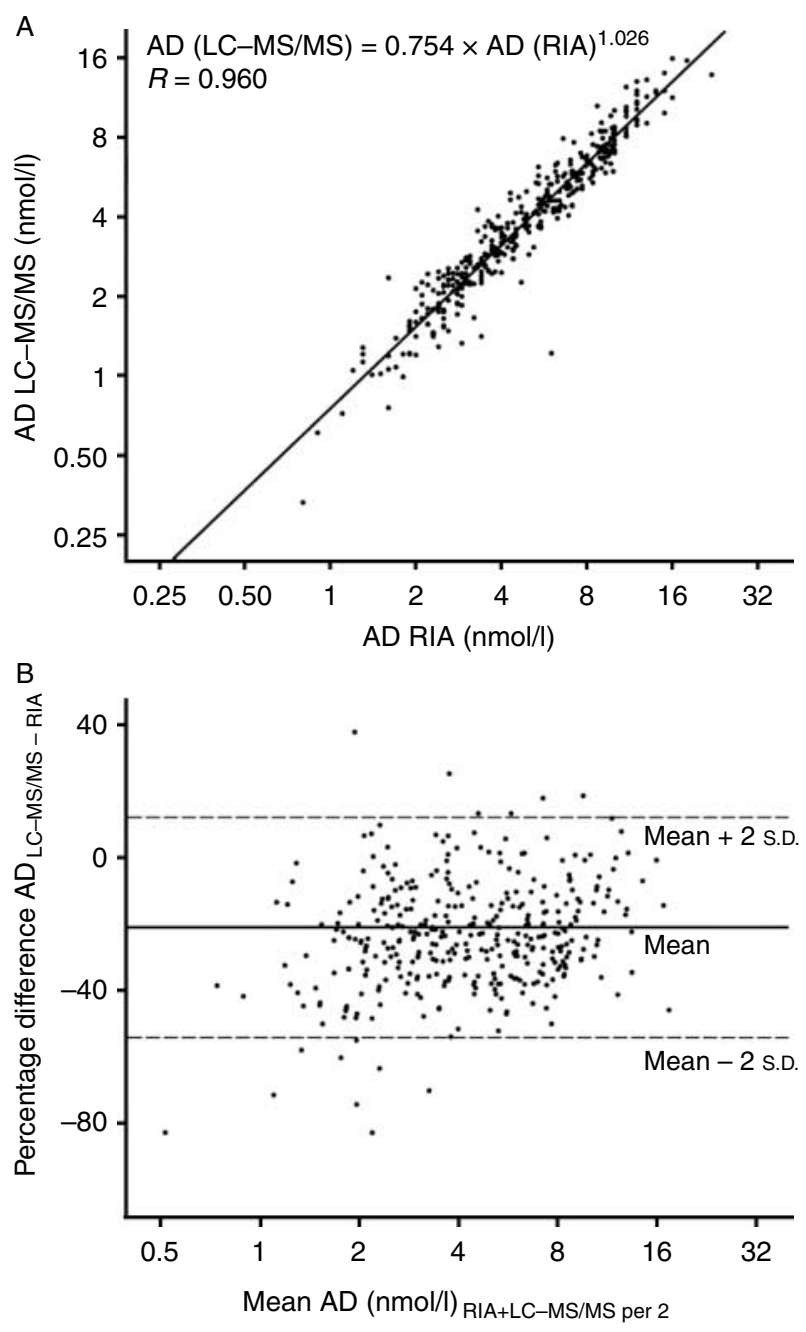

Figure 3 Scatter plot $(A)$ and Bland-Altman plot $(B)$ to show the extent to which $A D$ measurements agreed in both methods. The solid line represents the mean percentage difference between the methods (mean bias) and the dashed lines 2 s.D. of the mean percentage difference (limits of agreement). Filled circles represent individual measurements.

with LC-MS/MS should be performed to assess whether the advantage of the high-throughput character of the latter assay is still preferable. Extraction RIAs may be highly reliable when appropriately validated, but the method is time consuming and costly (11). Commercially available direct testosterone assays, although simple and convenient, are known for the general overestimation of steroid concentrations in women (36). Both LC-MS/MS and extraction RIA lack this bias in the lower concentration range, although the extraction RIA needs to be recalibrated against proper standards. Proper validation will reduce the number of false positives and therefore reduce unnecessary medical examinations. LC-MS/MS is a promising new, accurate, sensitive, and high-throughput method for the measurement of sex hormone levels.
With the demonstration that LC-MS/MS, compared with a labor-intensive RIA, exhibits good precision, sensitivity, and high accuracy for measuring female androgen concentrations, a convenient assay has become available for clinical and research purposes. Reproducibility of the LC-MS/MS between different LCMS/MS laboratories needs to be assessed to meet the performance criteria as is customary for new assays, before this assay will be adopted universally. The next phase in the introduction of LC-MS/MS should aim at obtaining highly true and precise reference values. These reference values could then be applied using other assays with same accuracy for normo-, hypo-, and hyperandrogenic women.

\section{Supplementary data}

This is linked to the online version of the paper at http://dx.doi.org/10. 1530/EJE-11-0482.

\section{Declaration of interest}

The authors declare that there is no conflict of interest that could be perceived as prejudicing the impartiality of the research reported.

\section{Funding}

This work was supported by an unrestricted research grant of National Health and Medical Research Committee (NHMRC), Australia, to T E Hickey and R J Norman.

\section{Acknowledgements}

We would like to thank Andrew Dinan at CPR Pharma Pty Ltd, Adelaide, for his assistance in the measurement of androgens by LCMS/MS. We would like to thank Dr Yvonne M van Kasteren (Medical Center Alkmaar) and Dr Peter A van Dop (Catharina Hospital, Eindhoven) for the inclusion of two and seven patients with POI.

\section{References}

1 Rosner W, Auchus RJ, Azziz R, Sluss PM \& Raff H. Position statement: utility, limitations, and pitfalls in measuring testosterone: an Endocrine Society position statement. Journal of Clinical Endocrinology and Metabolism 200792 405-413. (doi:10.1210/ jc.2006-1864)

2 Vermeulen A, Verdonck L \& Kaufman JM. A critical evaluation of simple methods for the estimation of free testosterone in serum. Journal of Clinical Endocrinology and Metabolism $1999 \mathbf{8 4}$ 3666-3672. (doi:10.1210/jc.84.10.3666)

3 Herold DA \& Fitzgerald RL. Immunoassays for testosterone in women: better than a guess? Clinical Chemistry $2003 \mathbf{4 9}$ 1250-1251. (doi:10.1373/49.8.1250)

4 Longcope C. Adrenal and gonadal androgen secretion in normal females. Clinics in Endocrinology and Metabolism 198615 213-228. (doi:10.1016/S0300-595X(86)80021-4)

5 Burger HG. Androgen production in women. Fertility and Sterility 200277 3-5. (doi:10.1016/S0015-0282(02)02985-0)

6 Dunn JF, Nisula BC \& Rodbard D. Transport of steroid hormones: binding of 21 endogenous steroids to both testosterone-binding globulin and corticosteroid-binding globulin in human plasma. Journal of Clinical Endocrinology and Metabolism 198153 58-68. (doi:10.1210/jcem-53-1-58) 
7 Stanczyk FZ, Cho MM, Endres DB, Morrison JL, Patel S \& Paulson RJ. Limitations of direct estradiol and testosterone immunoassay kits. Steroids 200368 1173-1178. (doi:10.1016/ j.steroids.2003.08.012)

8 Taieb J, Mathian B, Millot F, Patricot MC, Mathieu E, Queyrel N, Lacroix I, Somma-Delpero C \& Boudou P. Testosterone measured by 10 immunoassays and by isotope-dilution gas chromatography-mass spectrometry in sera from 116 men, women, and children. Clinical Chemistry 200349 1381-1395. (doi:10.1373/ 49.8.1381)

9 Boots LR, Potter S, Potter D \& Azziz R. Measurement of total serum testosterone levels using commercially available kits: high degree of between-kit variability. Fertility and Sterility 199869 286-292. (doi:10.1016/S0015-0282(97)00464-0)

10 Rosner $\mathrm{W} \&$ Vesper $\mathrm{H}$. Toward excellence in testosterone testing: a consensus statement. Journal of Clinical Endocrinology and Metabolism 201095 4542-4548. (doi:10.1210/jc.2010-1314)

11 Stanczyk FZ. Measurement of androgens in women. Seminars in Reproductive Medicine 200624 78-85. (doi:10.1055/s-2006939566)

12 Vesper HW, Botelho JC, Shacklady C, Smith A \& Myers GL. CDC project on standardizing steroid hormone measurements. Steroids 200873 1286-1292. (doi:10.1016/j.steroids.2008.09.008)

13 Shifren JL. Androgen deficiency in the oophorectomized woman. Fertility and Sterility 200277 60-62. (doi:10.1016/S00150282(02)02970-9)

14 van der Stege JG, Groen H, van Zadelhoff SJ, Lambalk CB, Braat DD, van Kasteren YM, van Santbrink EJ, Apperloo MJ, Weijmar Schultz WC \& Hoek A. Decreased androgen concentrations and diminished general and sexual well-being in women with premature ovarian failure. Menopause 200815 23-31. (doi:10.1097/gme.0b013e3180f6108c)

15 Davison SL, Bell R, Donath S, Montalto JG \& Davis SR. Androgen levels in adult females: changes with age, menopause, and oophorectomy. Journal of Clinical Endocrinology and Metabolism 200590 3847-3853. (doi:10.1210/jc.2005-0212)

16 Chang WY, Knochenhauer ES, Bartolucci AA \& Azziz R. Phenotypic spectrum of polycystic ovary syndrome: clinical and biochemical characterization of the three major clinical subgroups. Fertility and Sterility 200583 1717-1723. (doi:10.1016/ j.fertnstert.2005.01.096)

17 Rotterdam ESHRE/ASRM-sponsored PCOS consensus workshop group. Revised 2003 consensus on diagnostic criteria and longterm health risks related to polycystic ovary syndrome (PCOS). Human Reproduction 200419 41-47. (doi:10.1093/humrep/ deh098)

18 Bachmann G, Bancroft J, Braunstein G, Burger H, Davis S, Dennerstein L, Goldstein I, Guay A, Leiblum S, Lobo R, Notelovitz M, Rosen R, Sarrel P, Sherwin B, Simon J, Simpson E, Shifren J, Spark R \& Traish A. Female androgen insufficiency: the princeton consensus statement on definition, classification, and assessment. Fertility and Sterility 200277 660-665. (doi:10. 1016/S0015-0282(02)02969-2)

19 Goverde AJ, van Koert AJ, Eijkemans MJ, Knauff EA, Westerveld HE, Fauser BC \& Broekmans FJ. Indicators for metabolic disturbances in anovulatory women with polycystic ovary syndrome diagnosed according to the Rotterdam consensus criteria. Human Reproduction 200924 710-717. (doi:10.1093/ humrep/den433)

20 Janse F, Knauff EA, Niermeijer MF, Eijkemans MJ, Laven JS, Lambalk CB, Fauser BC \& Goverde AJ. Similar phenotype characteristics comparing familial and sporadic premature ovarian failure. Menopause 201017 758-765. (doi:10.1097/ gme.Ob013e3181cf8521)

21 Coulam CB, Adamson SC \& Annegers JF. Incidence of premature ovarian failure. Obstetrics and Gynecology $198667604-606$.

22 Knauff EAH, Eijkemans MJC, Lambalk CB, ten Kate-Booij MJ, Hoek A, Beerendonk CCM, Laven JSE, Goverde AJ, Broekmans FJM, Themmen APN, de Jong FH, Fauser BCJM \& on behalf of the Dutch Premature Ovarian Failure Consortium. Anti-Mullerian hormone, inhibin B, and antral follicle count in young women with ovarian failure. Journal of Clinical Endocrinology and Metabolism 2009 94 786-792. (doi:10.1210/jc.2008-1818)

23 Thienpont LM, Van Uytfanghe K, Blincko S, Ramsay CS, Xie H, Doss RC, Keevil BG, Owen LJ, Rockwood AL, Kushnir MM, Chun KY, Chandler DW, Field HP \& Sluss PM. State-of-the-art of serum testosterone measurement by isotope dilution-liquid chromatography-tandem mass spectrometry. Clinical Chemistry 200854 1290-1297. (doi:10.1373/clinchem.2008.105841)

24 Carter GD, Holland SM, Alaghband-Zadeh J, Rayman G, Dorrington-Ward P \& Wise PH. Investigation of hirsutism: testosterone is not enough. Annals of Clinical Biochemistry 198320 262-263.

25 Shrout PE \& Fleiss JL. Intraclass correlations: uses in assessing rater reliability. Psychological Bulletin 197986 420-428. (doi:10. 1037/0033-2909.86.2.420)

26 Stener-Victorin E, Holm G, Labrie F, Nilsson L, Janson PO \& Ohlsson C. Are there any sensitive and specific sex steroid markers for polycystic ovary syndrome? Journal of Clinical Endocrinology and Metabolism 2010 95 810-819. (doi:10.1210/jc.2009-1908)

27 Barth JH, Field HP, Yasmin E \& Balen AH. Defining hyperandrogenism in polycystic ovary syndrome: measurement of testosterone and androstenedione by liquid chromatographytandem mass spectrometry and analysis by receiver operator characteristic plots. European Journal of Endocrinology 2010162 611-615. (doi:10.1530/EJE-09-0741)

28 Helsel DR. Nondetects and Data Analysis: Statistics for Censored Environmental Data, edn 1. Eds M Scott \& V Barnett. Hoboken, NJ: John Wiley \& Sons, Inc., 2005.

29 Kalantaridou SN, Calis KA, Vanderhoof VH, Bakalov VK, Corrigan EC, Troendle JF \& Nelson LM. Testosterone deficiency in young women with $46, \mathrm{XX}$ spontaneous premature ovarian failure. Fertility and Sterility 200686 1475-1482. (doi:10.1016/ j.fertnstert.2006.04.028)

30 Benetti-Pinto CL, Bedone AJ \& Magna LA. Evaluation of serum androgen levels in women with premature ovarian failure. Fertility and Sterility 200583 508-510. (doi:10.1016/j.fertnstert.2004. 09.015)

31 Duignan NM, Shaw RW, Glass MR, Butt WR \& Edwards RL. Sex hormone levels and gonadotrophin release in premature ovarian failure. British Journal of Obstetrics and Gynaecology 1978 85 862-867. (doi:10.1111/j.1471-0528.1978.tb15844.x)

32 Falsetti L, Scalchi S, Villani MT \& Bugari G. Premature ovarian failure. Gynecological Endocrinology 199913 189-195. (doi:10. 3109/09513599909167554)

33 Bernardi F, Hartmann B, Casarosa E, Luisi S, Stomati M, Fadalti M, Florio P, Santuz M, Luisi M, Petraglia F \& Genazzani AR. High levels of serum allopregnanolone in women with premature ovarian failure. Gynecological Endocrinology 199812 339-345. (doi:10.3109/09513599809012836)

34 Elias AN, Pandian MR \& Rojas FJ. Serum levels of androstenedione, testosterone and dehydroepiandrosterone sulfate in patients with premature ovarian failure to age-matched menstruating controls. Gynecologic and Obstetric Investigation 199743 47-48. (doi:10. 1159/000291817)

35 Parker CR Jr, Slayden SM, Azziz R, Crabbe SL, Hines GA, Boots LR \& Bae S. Effects of aging on adrenal function in the human: responsiveness and sensitivity of adrenal androgens and cortisol to adrenocorticotropin in premenopausal and postmenopausal women. Journal of Clinical Endocrinology and Metabolism $2000 \mathbf{8 5}$ 48-54. (doi:10.1210/jc.85.1.48)

36 Wang C, Catlin DH, Demers LM, Starcevic B \& Swerdloff RS. Measurement of total serum testosterone in adult men: comparison of current laboratory methods versus liquid chromatographytandem mass spectrometry. Journal of Clinical Endocrinology and Metabolism 2004 89 534-543. (doi:10.1210/jc.2003-031287)

37 Moal V, Mathieu E, Reynier P, Malthiery Y \& Gallois Y. Low serum testosterone assayed by liquid chromatography-tandem mass spectrometry. Comparison with five immunoassay techniques. Clinica Chimica Acta 2007386 12-19. (doi:10.1016/j.cca.2007. 07.013) 
38 Owen WE, Rawlins ML \& Roberts WL. Selected performance characteristics of the Roche Elecsys testosterone II assay on the Modular analytics E 170 analyzer. Clinica Chimica Acta $2010 \mathbf{4 1 1}$ 1073-1079. (doi:10.1016/j.cca.2010.03.041)

39 Dorgan JF, Fears TR, McMahon RP, Aronson FL, Patterson BH \& Greenhut SF. Measurement of steroid sex hormones in serum: a comparison of radioimmunoassay and mass spectrometry. Steroids $2002 \quad 67$ 151-158. (doi:10.1016/S0039-128X(01) 00147-7)

40 Legro RS, Schlaff WD, Diamond MP, Coutifaris C, Casson PR, Brzyski RG, Christman GM, Trussell JC, Krawetz SA, Snyder PJ, Ohl D, Carson SA, Steinkampf MP, Carr BR, McGovern PG, Cataldo NA, Gosman GG, Nestler JE, Myers ER, Santoro N, Eisenberg E, Zhang M \& Zhang H. Total testosterone assays in women with polycystic ovary syndrome: precision and correlation with hirsutism. Journal of Clinical Endocrinology and Metabolism 201095 5305-5313. (doi:10.1210/jc.2010-1123)
41 Rivera-Woll LM, Papalia M, Davis SR \& Burger HG. Androgen insufficiency in women: diagnostic and therapeutic implications. Human Reproduction Update 200410 421-432. (doi:10.1093/ humupd/dmh037)

42 Mueller A, Cupisti S, Binder H, Hoffmann I, Kiesewetter F, Beckmann MW \& Dittrich R. Endocrinological markers for assessment of hyperandrogenemia in hirsute women. Hormone Research 200767 35-41. (doi:10.1159/000096036)

43 Bolelli G, Muti P, Micheli A, Sciajno R, Franceschetti F, Krogh V, Pisani P \& Berrino F. Validity for epidemiological studies of long-term cryoconservation of steroid and protein hormones in serum and plasma. Cancer Epidemiology, Biomarkers \& Prevention 1995 409-513.

Received 31 May 2011

Revised version received 28 September 2011

Accepted 3 October 2011 